\title{
Trismus-pseudocamptodactyly syndrome
}

INSERM

\section{Source}

INSERM. (1999). Orphanet: an online rare disease and orphan drug data base. Trismuspseudocamptodactyly syndrome. ORPHA:3377

A rare, genetic, distal arthrogryposis characterized by pseudocamptodactyly, mild foot deformities, moderately short stature, and short muscles and tendons resulting in a limited range of motion of the hands, legs, and mouth, the later presenting with trismus. 